\title{
Extended Service Oriented Architecture using Work Flow Management System and Petri Nets (ESOASCM)
}

\author{
Sujit Kr Singh \\ Ph.D Research Scholar \\ Mewar University, Chittorgarh Rajasthan
}

\author{
S Srinivasan, Ph.d \\ Professor \& Head C.S.A \\ P.D.M College of Engineering
}

\begin{abstract}
Intend to provide quick, efficient and effective sharing of resources across different organizations with the context of Supply Chain Management, Orchestration Engine and various web services are involved. With various technologies a model is proposed and the proposed model supports multiple applications of different organizations running on various platforms with seamless communication. In this research work ,the proposed model an Extended Service Oriented Architecture using Orchestration Engine for Supply Chain Management (ESOASCM) supports supply chain, consists of all parties involved, directly or indirectly, in fulfilling a customer request. The Work Flow Management System (WfMS),Service Oriented Architecture (S.O.A), Orchestration Engine are the major components of Extended Service Oriented Architecture .An Orchestration Engine for Supply Chain Management (ESOASCM) is designed and it is established how it helps the manufacturing unit. It has been argued how it reduces time of implementation, cost and managing inventory which is the major concern of any industry and builds a model to improve SCM performance in a manufacturing unit. All the services such as WfMS, Orchestration Engine (O.E), Petri-nets, and Supply Chain Management are designed integrated and architect separately and brought them together.
\end{abstract}

\section{Keywords}

Service oriented architecture, supply chain management, work flow management system, orchestration engine.

\section{INTRODUCTION}

In this research work, we propose an Extended Service Oriented Architecture using Orchestration Engine for Supply Chain Management (ESOASCM). To propose such model we involve various technologies.

\subsection{Service Oriented Architecture (S.O.A)}

A service-oriented architecture (SOA) [1] is an architectural style for combining software applications that utilize available services over a network. It is an area for organizing and utilizing distributed capabilities that may be under the control of different ownership domains. An SOA may support a variety of different communication protocols, but common protocols based on open standards (e.g., SOAP and WSDL) are used in general in SOA [2].

Implementations. These open standards are not the only technology with which an SOA can be established but it is likely that many SOAs which are large will also provide access to services with a combination of several technologies [3].

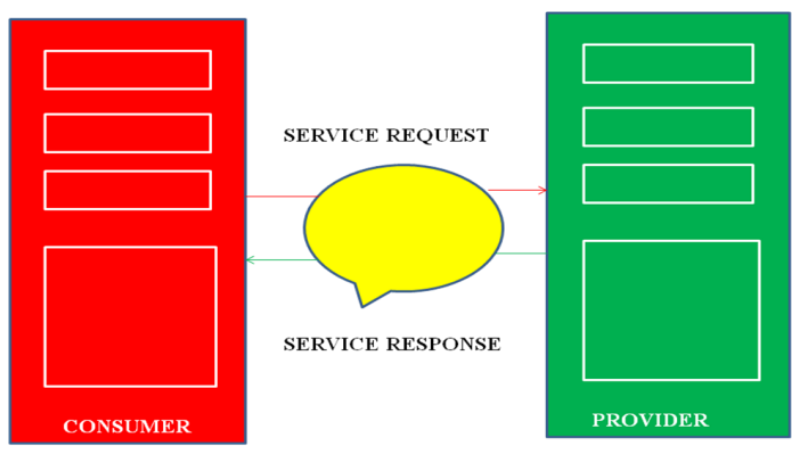

Fig 1: service oriented architecture

\subsection{Work Flow Management System (WfMS)}

Workflow is concerned with the automation of procedures where documents, information or tasks are passed between participants according to a defined set of rules to achieve, or contribute to an overall business goal [4]. WfMS improves organizational performance, decrease significantly the lead time, service and wait-time of business process execution, and increase the utilization of involved human resources. The main functions of a WfMS is to provide procedural automation of a business process, by managing of the sequence of work activities and the invoking of appropriate human or resources associated with the various activity steps [5].

\subsection{Petri nets}

Petri Nets (PN) are usually used for formal modeling of WFs due to its formal semantics, graphical description, and abundance of analysis techniques [6]. PN has been widely used for specifying, analyzing and verifying the properties of static workflow models.

\subsection{WFMS with Petrinet}

In WFMS development lifecycle, modeling is the first and most important stage and it lacks a standardized theory that provides a theoretical background. Petri Nets (PN) are used for modeling of WFs because of its formal semantics, graphical description. PN has been widely used for specifying the work flows, analyzing different paths and verifying the significant impact of work flow [7]. 


\subsection{Agent Based SOA SCM}

MAS-SOA-SCM [8] is based on the following hypotheses: 1. Cooperation and coordination among agents help information sharing which is associated with higher supply chain performance.

2. Less proactive which leads to complexity of the information sharing process, is associated with lower supply chain performance.

3. Collaborative approach of agents leads to positive information sharing transparency on supply chain performance is higher for firms with higher MAS-SOA adoption

4. Negative impact of information sharing on supply chain performance is lower for firms with higher MAS-SOA

At the manufacturer, MAS-SOA-SCM receives orders from customers and interacts with manager agent through order processing agent. Production agent fulfils the order through scheduling agent and delivery agent. In case of need more production, scheduling agent coordinates with inventory agent. Inventory agent takes care of supply of raw materials to production or collaborates with supplier agent for further negotiation with suppliers. Most importantly, MAS-SOASCM aims to meet the needs of the customers on time and to eliminate the need for a large inventory at the manufacturer and thus reduce overall cost in the entire manufacturing system. MAS based SOA strategy helps

Development process quick and deployment across the business.

\section{Fig: 2B Agent System based SOA-SCM Model}

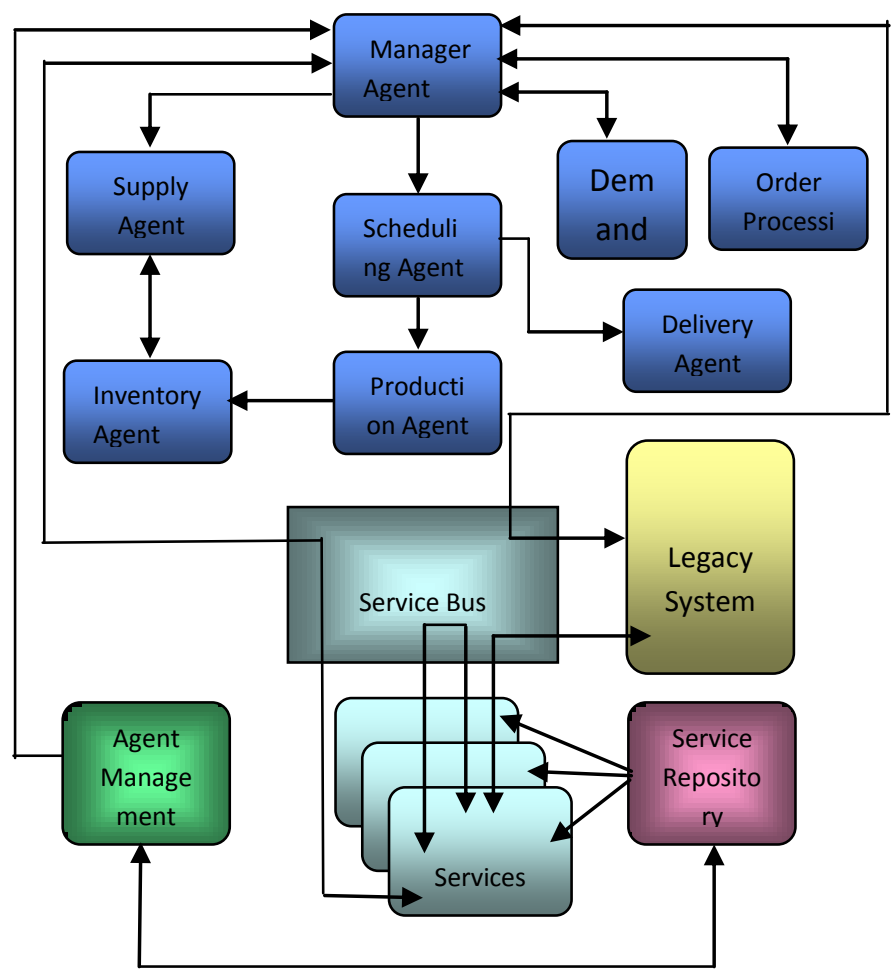

\section{SUPPLY CHAIN MANAGEMENT (SCM)}

A SUPPLY CHAIN [9] is a network of supplier, manufacturing, assembly, distribution, and logistics facilities that perform the functions of procurement of materials, transformation of these materials into intermediate and finished products, and the distribution of these products to customers. Supply chains arise in both manufacturing and service organization [10]

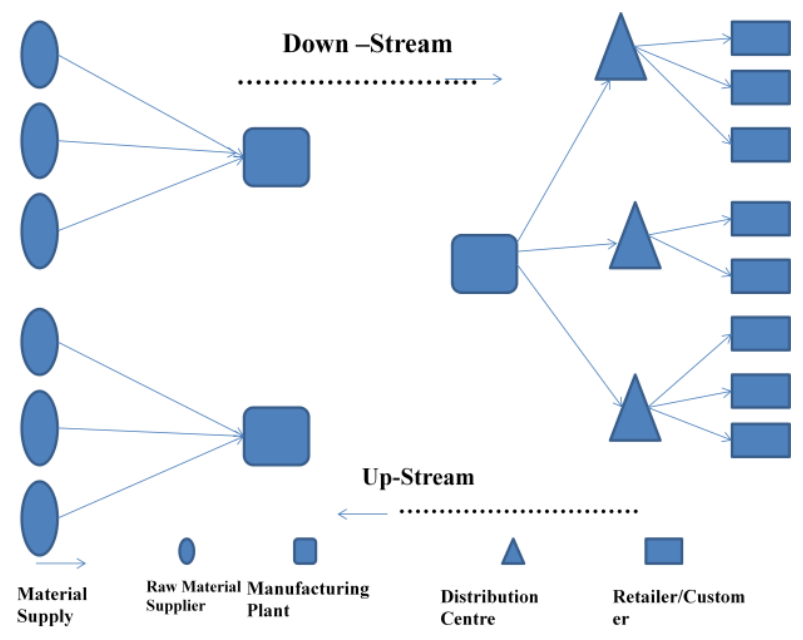

Fig: 2A Simple Petri nets for Supply Chain Management

Also supply chain management flows are made of three important flows. The product flow: This flow involves the movement of producers from a supplier to manufacturers to warehouse to a customer. The information flow: This flow consists of transmitting and updating the status of delivery. The financial flow: This flow contains credit terms, Payment details and schedules, and consignment

\subsection{Orchestration Engine}

Orchestration describes the automated arrangement, coordination, and management of a complex computer system with middleware and services. Orchestration is nothing but aligning the business request with the applications, data and infrastructure. It defines the policies and service levels through automated workflows, provisioning, and change management. Orchestration engine creates an applicationaligned infrastructure that can be scaled up or down based on the needs of each application. Orchestration also provides centralized management of the resource pool, including billing, metering, and chargeback for consumption [11]. For example, orchestration reduces the time and effort for deploying multiple instances of a single application. The orchestration engine proposed in our work handles multiple functions for multiple localities. As the requirement for more resources or a new application is triggered, automated tools perform tasks

\subsection{Orchestration Engine Based Supply Chain}

In Orchestration Engine based supply Chain Management we try to explain how a particular request from a manufacturer firm goes to Orchestration engine through Supply Chain Management and how such Request being processed with the help of toll box. 


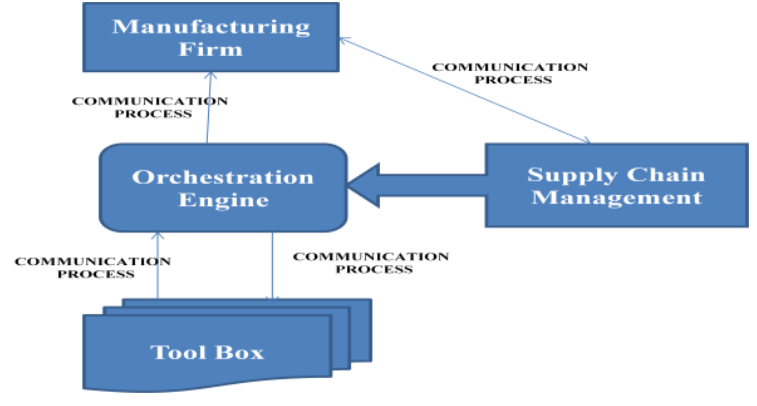

Fig 2B: SOA based SCM

Suppose manufacturing firm's production demand is high and it require several requests associated with the production then such request will be monitored by the Supply Chain Management (SCM) box. In such case SCM box will redesign the entire process of SCM adapted by the particular firm and based on the production request the SCM box will check the warehouse (total space occupied and free spaces available), production house( such as no of machine available at present moment and man power currently working in the firm), transportation for both the supplier and distributor ends. In order to fulfill such demands it will choreograph the entire request and send to the orchestration engine for the processing. Orchestration engine will takes the request and develop a Petri net model for each request and to process such request it will take the help of tool box which comprises of various web services. Orchestration engine will pick the suitable web services for the processing of the request. Once the request gets processed it will send back responses to the firm. All the request-response dispatcher between the Firm, SCM, Orchestration Engine and Tool box will be carried out by the help of Communication Process. Communication will be done by the help of XML (Extensible Markup Language), SOAP (Simple Object Access Protocols) and WSDL (Web Service description Language) [12].

\section{EXTENDED SOA WITH SUPPLY CHAIN MANAGEMENT USING ORCHESTRATION ENGINE (ESOASCM)}

ESOASCM consists of 3 major components namely Orchestration engine, SCM box and Tool box. ESOASCM simultaneously deals with many manufacturing units irrespective of what kind of products or business they are dealing with. SCM box monitors whether all the units work according to SCM procedures provided by SCM box. Tool box is a group of various web services and each web service deals with a particular type of program that is related to manufacturing. Supplier web service deals with problems related suppliers like supplier selection, delivery or delivery schedules of raw materials and so on. Orchestration engine is the main component and once it gets request from SCM box, it refers to tool box to select the appropriate services and using WFMS and Petri nets to stream line the activities and respond to the manufacturer and updates with SCM box. Fig. Represents the architecture of ESOASCM.

\subsection{Description of ESOASCM}

Step1: F1,F2, .....F3 are the respective manufacturing Industries it may be Computer Manufacturer, Automobile Industries, Electrical Equipment Industries etc...
They all have their respective orchestration engine as well as Petri Nets for their order request processing. It all connected with the main Orchestration Engine, Supply Chain Management and as well as Tool Box.

Step 1: Whenever F1,F2...Fn demand for any request like raw material supply, final product supply, Supply Chain Management(SCM) will look after(monitor) all these issues and the subsequent request will send by SCM to the Orchestration Engine(O.E).

Step 2: Supply Chain Management (SCM) box will continuously monitor the activities of the manufacturing units and whenever any request arises from Manufacturing Units it will pick up the request and convey it to the Orchestration Engine (O.E).Based on their need and request SCM box process model for different scenario. When there is the level of a particular component / raw materials falls below the minimum order level(M.O.L) -it is a quantity of raw materials fixed by the SCM) in any manufacturing unit the corresponding orchestration box senses it and send a request to SCM box which in turn forwards to Orchestration engine. It will turn to tool box to fix the problem.Orchestartion engine picks the relevant web service or series of web services and with the help of WFMS and Petrinet, design the flow model and pass it on to both respective orchestration box and SCM box. This working model of supplier selection Process model will be kept in the memory of orchestration engine for future purpose. If the similar request comes from SCM box, Orchestration engine picks up from its memory box. Orchestration keeps the record of all the activities similar to Case Base Reasoning System.

Step 3: Orchestration Engine (O.E): It comprises of Work flow management system (WFMS) model and Petri Nets along with the Memory Unit for storage of subsequent request and response arises from the SCM and Tool Box.

Step 3: Orchestration Engine (O.E): It comprises of Work flow management system (WFMS) model and Petri Nets along with the Memory Unit for storage of subsequent request and response arises from the SCM and Tool Box.

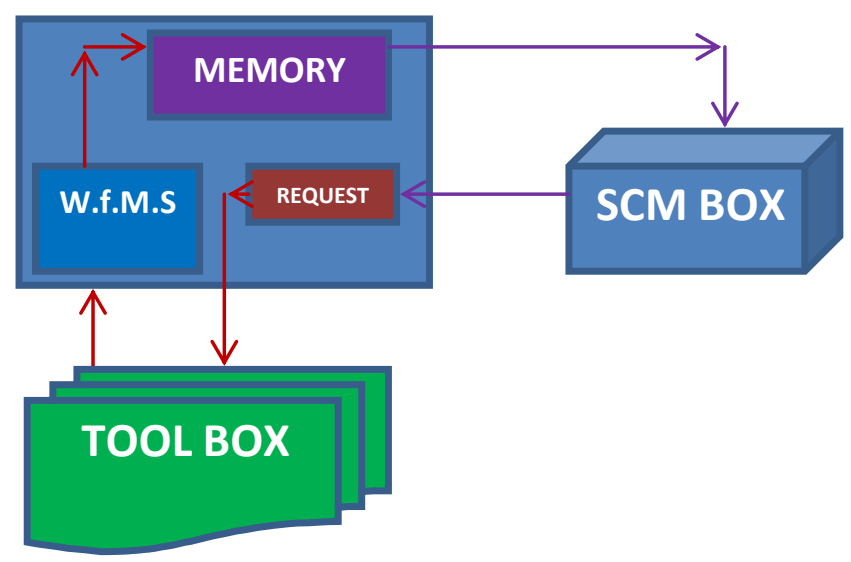

Fig3A: Architecture of Orchestration Engine

\subsection{Role of Orchestration Engine}

Orchestration engine will process the request (e.g. suppose the request is for raw materials supplier) it will generate a negotiation model based selection criteria for suitable supplier. Selection for a suitable supplier will be done based on QPT (Quality Price Time) Analysis Techniques. Such 
Supplier will be chosen who deliver better Quality in minimum cost and time.

\subsection{Memory Box}

It is a part of Orchestration engine. Whenever it receives a request SCM box, it looks for a solution with in itself. It keeps record of all the issues that comes as a request from SCM box and also the subsequent responses from the Tool Box. Whenever it gets the request for the first time orchestration engine will refer to the toll box to find the web services, using WFMS to find the flow of services and with the help of Petrinet to standardize the flow and forward it as responses to SCM box. At the same time the response for the current request will be kept in the memory unit. When the Orchestration engine receives the same request, memory box identifies the request and it responds to SCM box without referring to tool box. It works exactly similar to Case Based Reasoning System.

Tool Box: It comprises of various web services (SOA) designed based on XML, WSDL and SOAP.

SOAP pertaining to SCM functions. Web Service will provide the suitable solution for the request and allows the orchestration engine to select.

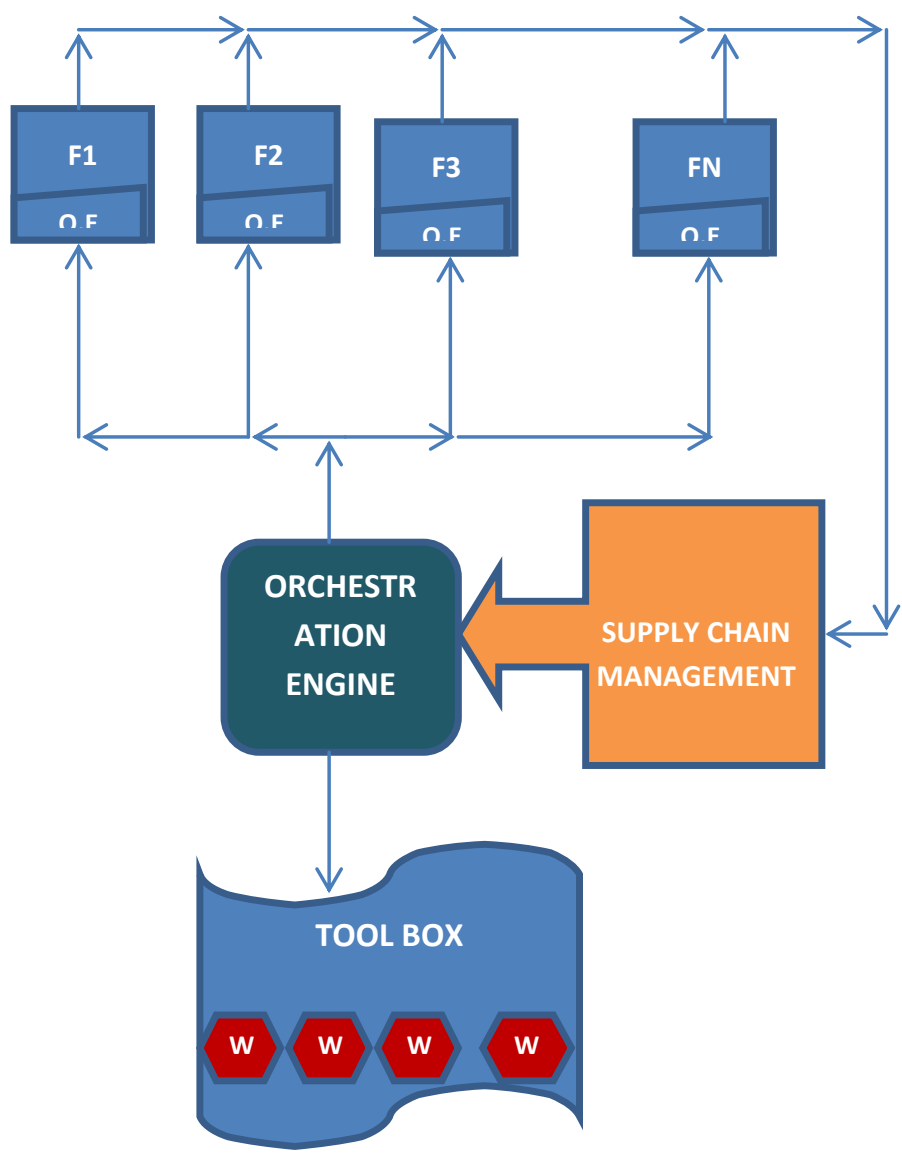

Fig 3B: Extended Service Oriented Architecture for supply chain management

\section{CONCLUSION}

The proposed model ESOASCM works on different platforms irrespective of the nature of various manufacturing units. It provides a right Supply Chain Management (SCM) system to any kind of manufacturing industry and orchestration Engine (O.E) with the help of various Web Services an efficient way of SCM in context of enhancing the manufacturing of materials in lieu of time, quality \& budget. Our future intention is to introduce agent technologies to reduce the complexity of this model (ESOASCM) and also make such model effective in Customer Relationship Management (CRM)[13] areas.

\section{ACKNOWLEDGMENTS}

Our thanks to the experts who have contributed towards development of the template.

\section{REFERENCES}

[1] Service Oriented Architecture (SOA) Based Web Services For Geographic Information Systems K. Sahina, *, M. U. Gumusayb.

[2] Service Oriented Architecture Adoption: Key Factors and Approaches Journal of Information Technology Managementby Andrew P Ciganek \& William D. Haseman

[3] The Composition Approach for Large-Scale SOA http://www.tibco.com/resources/solutions/soa/pushtotest _soa_wp.pdf.

[4] Integrative Document and Content Management: Strategies for Exploiting By Len Asprey, Michael Middleton.

[5] A Prototype Workflow Engine Partially Supporting Yawl YI Chen Department of Computer Science \& Software Engineering Montreal Quebec Canada.

[6] Multilevel Petri Nets for the specification and development of Workflow Automation systems Marina Flores-Badillo and Ernesto Lopez-Mellado CINVESTAV Unidad Guadalajara Mexico

[7] Modeling and Simulation of Workflow Processes Using Multi-level Petri Nets Marina Flores-Badillo, Ernesto López-Mellado, Mayra Padilla-Duarte CINVESTAV Unidad Guadalajara.

[8] Multi-agent System based Service Oriented Architecture for Supply Chain Management System (MAS-SOASCM) By Sujit Kumar Singh and Dr S Srinivasan.

[9] Supply Chain Management-Knowledge Domain ChiaChin Cheng

[10] SupplyChain Management http: //www.eccouncil.org/docs/SCM.pdf

[11] Constructing an Orchestration Engine for Supply Chain Management system By Surjeet Dalal Dr. S Srinivasan

[12] Service-Oriented Architecture: Concepts, Technology \& Design by Thomas Erl. 